\title{
Simple linear measurements of the normal liver: Interobserver agreement and correlation with hepatic volume on MRI
}

\author{
Sachit K. Verma \\ Thomas Jefferson University \\ Kristen McClure \\ Thomas Jefferson University \\ Laurence Parker \\ Thomas Jefferson University \\ Donald G. Mitchell \\ Thomas Jefferson University

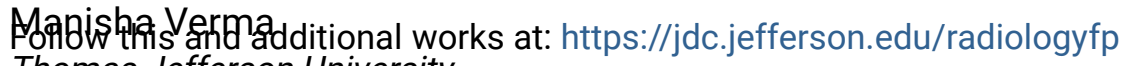 \\ Thomas Jefferson University \\ Part of the Medical Sciences Commons, and the Radiology Commons \\ Let us know how access to this document benefits you
}

\section{Recommended Citation}

Verma, Sachit K.; McClure, Kristen; Parker, Laurence; Mitchell, Donald G.; Verma, Manisha; and

Bergin, Diane, "Simple linear measurements of the normal liver: Interobserver agreement and correlation with hepatic volume on MRI" (2010). Department of Radiology Faculty Papers. Paper

8.

https://jdc.jefferson.edu/radiologyfp/8

This Article is brought to you for free and open access by the Jefferson Digital Commons. The Jefferson Digital Commons is a service of Thomas Jefferson University's Center for Teaching and Learning (CTL). The Commons is a showcase for Jefferson books and journals, peer-reviewed scholarly publications, unique historical collections from the University archives, and teaching tools. The Jefferson Digital Commons allows researchers and interested readers anywhere in the world to learn about and keep up to date with Jefferson scholarship. This article has been accepted for inclusion in Department of Radiology Faculty Papers by an authorized administrator of the Jefferson Digital Commons. For more information, please contact: JeffersonDigitalCommons@jefferson.edu. 
Authors

Sachit K. Verma, Kristen McClure, Laurence Parker, Donald G. Mitchell, Manisha Verma, and Diane Bergin 


\title{
Simple linear measurements of the normal liver: interobserver agreement and correlation with hepatic volume on MRI
}

\author{
Sachit K. Verma ${ }^{\mathbf{a}}$, Kristen McClure ${ }^{\mathbf{a}}$, Donald G. Mitchell ${ }^{\mathbf{a}}$, Laurence Parker ${ }^{\mathbf{a}}$, Manisha Verma ${ }^{\mathbf{a}}$ and Diane \\ $\operatorname{Bergin}^{\mathbf{a}, \mathbf{b}}$ \\ a Department of Radiology, Thomas Jefferson University Hospital, Philadelphia, PA, USA \\ ${ }^{\mathbf{b}}$ Current address: Department of Radiology, Galway University Hospital, Galway, Ireland.
}

\section{Introduction}

Estimation of liver size can be used as an index to monitor various aspects of liver disease and response to treatment [1, 2] Serial magnetic resonance imaging (MRI) may be used to monitor patient treatment and determine management [3]. Midclavicular (MCL), craniocaudad (CC), or midhepatic (MHP) CC measurements have been used in ultrasound (US) to estimate liver size [4, 5]. These methods have been extrapolated to advanced imaging modalities, including computed tomography (CT) and MRI [6-9].

There are no studies correlating simple linear hepatic measurements on MRI and hepatic volume. The aim of the present study was to determine interobserver agreement for the following linear hepatic measurements: MHP CC, maximum CC to liver tip (Max CC), maximum transverse, and MHP anteroposterior (AP) dimensions. Individual liver measurements and their products (Max CC and MHP CC by AP) were correlated with hepatic volume.

\section{Materials and methods}

\section{Patients}

This retrospective study was compliant with the Health Insurance Portability and Accountability Act and was approved by our institutional review board, who waived the requirement for informed patient consent. The hospital radiology information system was retrospectively searched to select patients who had undergone contrast enhanced abdominal MRI for conditions unrelated to the hepatobiliary system between December 2006 and September 2007. All patients had normal liver function. The final study group consisted of 116 patients (40 men, 76 women; age range 16-89, mean; 55.5 years).

\section{MRI technique}

All MR exams were performed during suspended respiration with a 1.5-T system and a phasedarray coil (Signa; GE Medical Systems, Milwaukee, Wis). The sequences included twodimensional coronal and transverse single-shot fast spin-echo T2-weighted MR imaging (echo time, 180-200 $\mathrm{msec}$ ), transverse fat-suppressed fast spin-echo T2-weighted MR imaging (repetition time msec/echo time msec, 2000-4000/70-90), and spoiled dual gradient-echo T1weighted in- and out-of-phase MR imaging (120-200/2.3 and 4.6, 90 flip angle). Parameters for two-dimensional images included 5-8-mm-thick sections with a 1-mm intersection gap, $256 \mathrm{x}$ 
160-192 matrix, $32 \times 24 \mathrm{~cm}^{2}$ field of view. Three-dimensional (3D) dynamic contrast-enhanced spoiled dual gradient-echo dynamic fat-suppressed MR images were obtained at 2.5-mm increments by using zerofill interpolation, 6.1/2.1, and $12^{\circ}-20^{\circ}$ flip angle. Twenty milliliters of intravenous contrast (Magnevist; Berlex Laboratories, Wayne, NJ) was administered with a power injector (Optistar LE; Mallinckrodt, Hazelwood, Mo) at $2 \mathrm{~mL} / \mathrm{sec}$. This was followed by a 20-mL saline solution flush. First-pass arterial enhancement was optimized by using a timing bolus sequence or by observing enhancement on images reconstructed in real time. Dynamic imaging was performed during breath holding before the injection (unenhanced), immediately after the injection (hepatic arterial phase), 60-70 seconds after the injection (portal venous phase). Additional images of the entire liver were acquired in the delayed phase with a twodimensional single-section spoiled dual gradient-echo technique with 19-20/1.5-2.1 and a flip angle of $40^{\circ}$. Hepatic linear measurements may have been performed on any sequence, but for standardization, portal venous enhanced 3D gradient echo images were utilized.

\section{Linear hepatic measurements}

MRI data sets were transferred to a dedicated 3D workstation (Vitrea 2, Vital Images, Minnetonka, Minnesota, USA). Two experienced radiologists (S.K.V., D.B) independently evaluated the MRI images. The following measurements of the liver were performed independently by readers (1). Midhepatic point craniocaudad (MHP CC); (2). Maximum CC to liver tip (Max CC); (3). Maximum transverse dimension and (4). MHP anteroposterior (AP) dimension of the liver. The plane of the horizontal component of the main portal vein was identified and used as a reference point for measurements. The MHP was defined as half way between the mid vertebra and right lateral margin of the liver at the level of main portal vein on a transverse section (Fig. 1). MHP CC was defined as a perpendicular measurement on the coronal images from the hepatic dome to the inferior margin of the liver passing through the midhepatic point (Fig. 2). The Max CC was defined as the greatest obtainable craniocaudad dimension of the liver from the hepatic dome to the liver tip on coronal or sagittal reconstructed images (Fig. 2). Maximum transverse dimension was the maximum measurement from the right to left margins of the liver at the level of the portal vein (Fig. 3). MHP AP measurement was taken at the level of the midhepatic point from anterior to posterior margin of the liver (Fig. 3).

\section{Hepatic volume measurement}

Hepatic volume measurements were performed by a radiologist (S.K.V) experienced in volume measurements and blinded to linear hepatic measurements. Hepatic volume measurements were performed by tracing the contours of the liver on sequential $5 \mathrm{~mm}$ axial images. For consistency and because of best demonstration of the portal vein, volumetric measurements were also acquired on the portal venous phase images. Manual tracing was performed on all transverse images starting from the superior margin of the liver to the inferior margin in a series similar to algorithm proposed by Heymsfield et al [10]. Extraparenchymal blood vessels such as the inferior vena cava, portal vein and fissure for ligamentous teres were excluded. The gallbladder was also excluded. Intrahepatic veins and intraparenchymal inferior vena cava were included. A 3D volume dataset of the liver with calculated hepatic volume was generated from compilation of traced hepatic contour on sequential transverse images. 


\section{Statistical Analysis}

Single linear measurements and hepatic volume measurements were recorded. Products of linear measurements MHP CC x MHP AP and Max CC x MHP AP were determined Statistical analysis were conducted using SAS V. 9.13 for Windows (2006; SAS Institute, Gary, NC, USA). Descriptive statistics (mean, SD) were provided where appropriate. Linear hepatic measurements: MHP CC, Max CC, maximum transverse, MHP AP dimensions and products of both CC measurements with MHP AP dimension were compared with hepatic volumes by using Pearson product moment correlation coefficient (Pearson's $r$ ). Student's $t$ test was used to calculate statistical significance. A $p$ value of less than .05 was statistically significant. The intraclass correlation coefficient (ICC) of the two reader's measurements was determined by using the Shrout-Fliess Random test [11]

\section{Results}

\section{Linear hepatic and volume measurements}

Linear hepatic dimensions (expressed as means \pm standard deviations), as determined by two observers are summarized in Table 1. Product of MHP CC with MHP AP dimensions ranged from 79.70 to $312.87 \mathrm{~cm}^{2}$ (mean $183.13 \mathrm{~cm}^{2} \pm 47.07 \mathrm{~cm}^{2}$ ) and of Max CC with MHP AP dimensions ranged from 98.03 to $467.99 \mathrm{~cm}^{2}$ (mean $\left.265.58 \pm 68.26 \mathrm{~cm}^{2}\right) .113(96 \%)$ patients had MHP CC dimension of $16 \mathrm{~cm}$ or less (mean $12.2 \mathrm{~cm}$; range, 7.1-16 cm). 33 (28\%) patients had Max CC dimension of $16 \mathrm{~cm}$ or less (mean $14.8 \mathrm{~cm}$; range, 13.8-16 cm). The volume of liver ranged from 533 to $2417 \mathrm{~cm}^{3}$ (mean $1106 \pm 392 \mathrm{~cm}^{3}$ ).

\section{Relationship between linear hepatic measurements and volume}

There was a positive correlation between hepatic volume and linear hepatic measurements; MHP CC $(r=0.44, p<0.0001)$, Max CC $(r=0.51, p<0.0001)$ and MHP AP $(r=0.53, p<0.0001)$. There was no correlation between hepatic volume and maximum transverse measurement $(r=0.15, p=0.09)$.

There was a positive correlation between hepatic volume and product of MHP CC with MHP AP dimension $(r=0.78, p<0.0001)$ (Fig. 5) and product of Max CC with MHP AP dimension $(r=$ $0.68, p<0.0001)$ (Fig. 6).

\section{Interobserver agreement assessment}

There was an excellent interobserver agreement (ICC range 0.89 to 0.95 ) between readers for all linear hepatic measurements.

\section{Discussion}

Comprehensive US and radionuclide studies has been done to assess liver size, limited by poor resolution, body habitus, respiratory movements, field of view, poor acoustic window and observer dependency [5, 12-15] Cadaver studies have determined the reliability of sonographic 
measurement of the liver in the right MCL plane as an indicator of liver size [16, 17], but we are not aware of any criteria to define the liver size by linear measurements from CT or MRI. Measuring hepatic volume may be time consuming and software dependent. Therefore, for a busy clinical radiology practice, it is useful to have a simplified measuring method by which one can reliably compare liver size on comparative studies.

In this study four separate linear liver measurements were assessed as indicators of liver size. MHP was described by Gosink and Leymaster [16] as an index hepatic point on US to determine CC hepatic measurement.

The results of the present study show a good correlation between single linear measurements (MHP CC, Max CC and MHP AP) of the liver and hepatic volume (Pearson $r, 0.44,0.51$ and 0.53). It has been well documented that the product of the two longest diameter for tumor volume even though non perpendicular reduces the risk of potential bias [18]. In the present study there was excellent correlation between hepatic volume and products of the single measurements (MHP CC x MHP AP) or (Max CC x MHP AP) are used (Pearson $r$; 0.68 and $0.78)$. The present study validates the use of single linear hepatic measurements or their product as reliable and fast methods for estimating liver size. High interobserver agreement between our readers for linear measurements indicated reproducibility of these measurements.

On longitudinal sections, we evaluated two alternative liver dimensions; MHP CC and Max CC. Max CC is easier to measure, but this measurement may potentially be less reliable in the presence of tongue like projection known as Reidel's lobe arising from the right lobe of the liver. Max CC was found to be at least as good an indicator of hepatic size as MHP CC (Pearson $r$; Max CC 0.51 and MHP 0.44) in the present study. Kratzer et al [19] suggested liver length greater than $16 \mathrm{~cm}$ as criteria of defining hepatomegaly on US. $11.5 \%$ of patients in their study group exceeded $16 \mathrm{~cm}$. In the present study, only 4\% (5/116) patients had MHP CC greater than $16 \mathrm{~cm}$, confirming the present cohort of patients with healthy livers.

We are not aware of any consensus for defining normal hepatic volume by cross-sectional imaging. A recent study reported hepatic volumes (mean $1186 \mathrm{~cm}^{3}$, range; $639.3-2359.4 \mathrm{~cm}^{3}$ ) similar to ours study (mean $1106 \mathrm{~cm}^{3}$, range; 533-2417 $\mathrm{cm}^{3}$ ) of normal healthy livers [20]. Performing individual orthogonal liver measurements to assess liver size is a practical and simple indicator of liver size in a busy clinical radiology practice. Hepatic linear measurements may be obtained on any picture archiving and communication systems (PACS) without the need of additional image processing or a 3D workstation, and are readily comparable between serial studies of the same patient.

The present method of utilizing individual liver linear measurements in normal liver patients is validated by high correlation with the hepatic volume parameters and excellent interobserver agreement. Future studies will be necessary to validate these measurements in diseased livers. Although MRI images were used for measurement, it is anticipated that similar results would be obtained if CT rather than MRI are used to measure liver size using the same measurement techniques.

In conclusion, the present study validates single hepatic measurements; MHP CC, Max CC and MHP AP dimensions and their products as good indicators of hepatic size and a reliable method of comparing liver size on serial studies. Both $\mathrm{CC}$ measurements had similar correlation with hepatic volume. Max CC measurement of liver size to liver tip and MHP CC hepatic dimensions are easy and practical measurement methods for routine use. Thus linear hepatic measurements and their products can be reliably used to indicate hepatic size on MRI 


\section{References}

1. Gao L, Heath DG, Kuszyk BS, et al. Automatic liver segmentation technique for threedimensional visualization of CT data. Radiology 1996;201:359-64.

2. Strunk H, Stuckmann G, Textor J, et al. Limitations and pitfalls of couinaud's segmentation of the liver in transaxial imaging. Eur Radiol 2003;13:2472-82.

3. Ito K, Mitchell DG, Hann HW et al. Progressive viral-induced cirrhosis: serial MR imaging findings and clinical correlation. Radiology 207:729-35.

4. Naylor CD, McCormack DG, Sullivan SN. The midclavicular line: the midclavicular line: a wandering landmark. Can Med Assoc J 1987;136:48-50.

5. Leung NW, Farrant P, Peters TJ. Liver volume measurement by ultrasonography in normal subjects and alcoholic patients. J Hepatol 1986;2:157-64.

6. Emirzeoglu M, Sahin B, Selcuk M, et al. The effects of section thickness on the estimation of liver volume by the cavalieri principle using computed tomography images. Eur J Radiol 2005;56:391-7.

7. Cheng YF, Chen CL, Huang TL, et al. Single imaging modality evaluation of living donors in liver transplantation: magnetic resonance imaging. Transplantation 2001;72:1527-33.

8. Breiman RS, Beck JW, Korobkin M, et al. Volume determinations using computed tomography. AJR Am J Roentgenol 1982;138:329-33.

9. McNeal GR, Maynard WH, Branch R, et al. Liver volume measurements and threedimensional display from MR images. Radiology 1988;169:851-4.

10. Heymsfield SB, Fulenwider T, Nordlinger B, et al. Accurate measurement of liver, kidney, and spleen volume and mass by computerized axial tomography. Ann Intern Med 1979;90: 1857 .

11. Shrout PE, Fleiss JL. Intraclass correlations: uses in assessing rater reliability. Psychol Bull 1979;86:420-9.

12. Hermoye L, Laamari-Azjal I, Cao Z, et al. Liver segmentation in living liver transplant donors: comparison of semiautomatic and manual methods. Radiology 2005;234:171-8.

13. Nakayama Y, Li Q, Katsuragawa S, et al. Automated hepatic volumetry for living related liver transplantation at multisection CT. Radiology 2006;240:743-8.

14. Rosenfield AT, Schneider PB. Rapid evaluation of hepatic size on radioisotope scan. J Nucl Med 1970;11:237-40. 
15. Rollo FD, DeLand FH. The determination of liver mass by radionuclide images. Radiology 1968;91:1191-4.

16. Gosink BB, Leymaster CE. Ultrasonic determination of hepatomegaly. J Clin Ultrasound $1981 ; 9: 37-44$.

17. Raeth U, Johnson PJ, Williams R. Ultrasound determination of liver size and assessment of patients with malignant liver disease. Liver 1984;4:287-93.

18. Monsky WL, Raptopoulos V, Keogan MT, et al. Reproducibility of linear tumor measurements using PACS: comparison of caliper method with edge-tracing method. Eur Radiol 2004;14:519-25.

19. Kratzer W, Fritz V, Mason RA, et al. Roemerstein study Group. Factors affecting liver size: a sonographic survey of 2080 subjects. J Ultrasound Med 2003;22:1155-61.

20. Chandramohan A, Eapen A, Govil S, et al. Determining standard liver volume: assessment of existing formulae in Indian population. Indian J Gastroenterol 2007;26:22-5. 
Figures

Figure 1

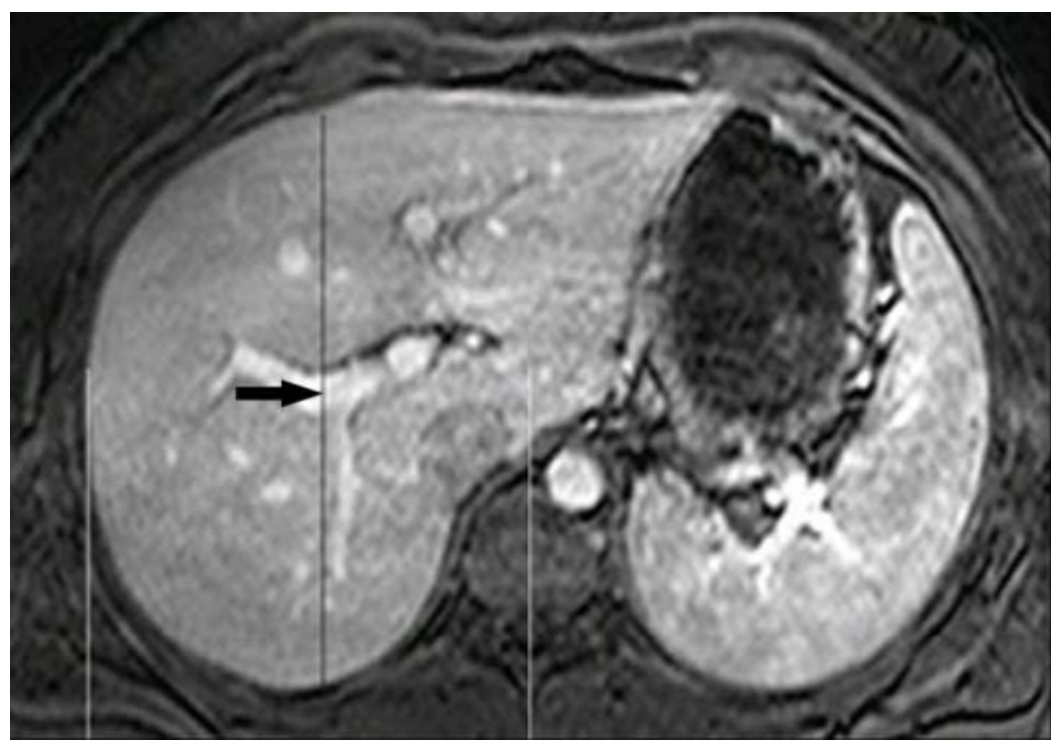

Figure 2

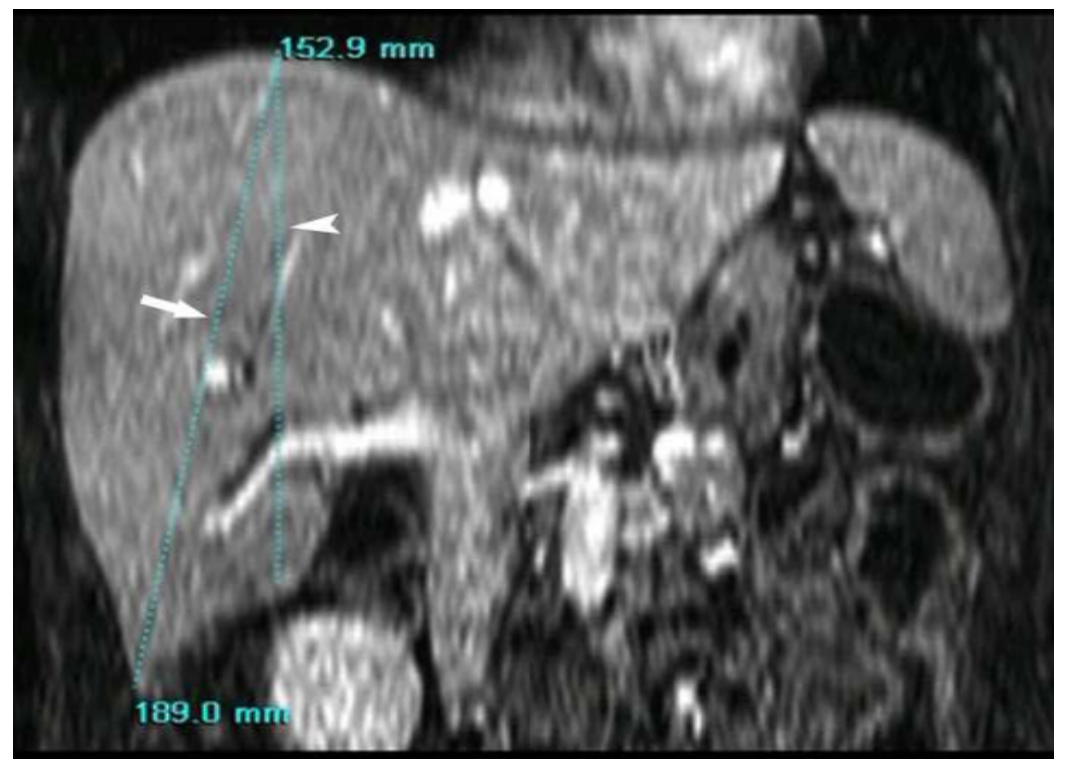


Figure 3

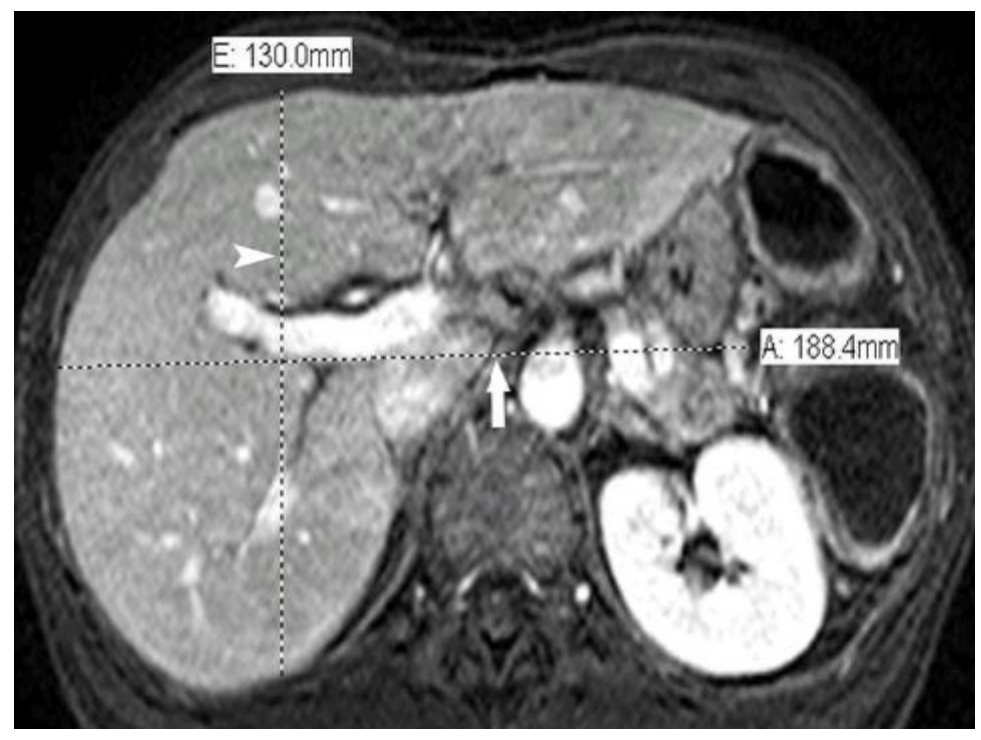


Table 1: Linear Dimensions of the Liver

\begin{tabular}{|l|l|}
\hline Dimension & Measurement $^{\mathbf{a}}(\mathrm{cm})$ \\
\hline MHP CC & $7.1-18.3(12.4 \pm 2.3)$ \\
Max CC & $13.8-24.9(17.8 \pm 2.3)$ \\
Maximum transverse & $10.6-25.3(18.4 \pm 2.6)$ \\
MHP AP & $6.2-20(14.8 \pm 2.7)$ \\
\hline MHP, mid hepatic point; Max, maximum; CC, craniocaudad; AP, anteroposterior. \\
a Data are range intervals and its mean diameter \pm standard deviations in the parenthesis. \\
\hline
\end{tabular}




\section{Figure Legends}

Figure 1-Axial, T1-weighted, 3D, gradient-echo MRI (TR/TE, 3.9/1.9 ms) in a 47-year-old woman shows mid-hepatic point (black arrow) half way between the mid vertebra and right lateral margin of the liver (white lines).

Figure 2-Coronal, T1-weighted, 3D, gradient-echo MRI (TR/TE, 3.9/1.9 ms) in a 47- year-old woman illustrates the method of depicting the midhepatic point craniocaudad (MHP CC) and maximum CC (Max CC) lengths of the liver. MHP CC is measured as the greatest overall dimension (arrowhead) and Max CC as the longest distance between superior to inferior-most tip of the liver (arrow).

Figure 3-Axial T1-weighted, 3D, gradient-echo MRI (TR/TE, 3.9/1.9 ms) in a 47-year-old woman illustrates the maximum transverse and midhepatic point anteroposterior (MHP AP) lengths of the liver. The maximum transverse dimension is measured as maximum distance between left and right lateral liver margins (arrow) and the MHP AP as the maximum AP dimension of the liver (arrowhead). 